\title{
Measurement of differential top-quark-pair production cross sections in the lepton+jets channel with CMS
}

\author{
Jörn Lange* on behalf of the CMS Collaboration \\ Institut für Experimentalphysik, Universität Hamburg, Germany \\ E-mail: joern. lange@cern.ch
}

\begin{abstract}
A measurement is presented of normalised differential top-quark-pair cross sections in the lepton+jets decay channel (e+jets and $\mu+j e t s)$. The analysis is based on data of pp collisions at a centre-of-mass energy of $8 \mathrm{TeV}$ at the LHC recorded by the CMS experiment in 2012, corresponding to $12.1 \mathrm{fb}^{-1}$. The cross sections are measured as a function of kinematic variables of the $t \bar{t}$ system, the top quarks and their decay products (charged leptons and $b$ jets). The results are compared to standard-model predictions from Monte-Carlo event generators and approximate next-to-next-to-leading-order calculations. A good description of the data is observed.
\end{abstract}

The European Physical Society Conference on High Energy Physics -EPS-HEP2013

18-24 July 2013

Stockholm, Sweden

\footnotetext{
* Speaker.
} 


\section{Introduction}

The large centre-of-mass energy and luminosity at the LHC allow for a detailed investigation of top-quark-pair $(\mathrm{t} \overline{\mathrm{t}})$ production properties, including differential cross sections. Such distributions are a test of the standard model (SM), especially of perturbative $\mathrm{QCD}$, and allow to constrain QCD parameters such as parton-distribution functions (PDFs). Furthermore, they are also relevant for scenarios beyond the SM because firstly $t \bar{t}$ events often constitute a major background in these searches and secondly these distributions are sensitive to potential new physcis effects themselves.

Already at a centre-of-mass energy of $\sqrt{s}=7 \mathrm{TeV}$, using data up to $5 \mathrm{fb}^{-1}$, first measurements $[3,4,5]$ of normalised differential $t \bar{t}$ cross sections have been performed by the CMS [1] and ATLAS [2] experiments. Here, an update of the measurement by CMS in the lepton+jets decay channel is presented, using data of pp collisions at a centre-of-mass energy of $8 \mathrm{TeV}$ at the LHC recorded in 2012, corresponding to $12.1 \mathrm{fb}^{-1}$. More details of this analysis are presented in Ref. [6]. A complementary measurement in the dileptonic decay channel is presented elsewhere [7].

\section{Event Selection, Reconstruction and Cross-Section Determination}

The measurement is based on a high-purity $\mathrm{t} \overline{\mathrm{t}}$-event selection according to the decay topology in the lepton+jets channel. Single isolated electron or muon triggers are used. Events are required to have exactly one well-isolated and identified electron or muon with a transverse momentum of $p_{\mathrm{T}}>30 \mathrm{GeV}$ and a pseudorapidity range of $|\eta|<2.1$ and at least four jets with $p_{\mathrm{T}}>30 \mathrm{GeV}$ and $|\eta|<2.4$, two out of which are required to be identified as originating from $b$ quarks. This results in $39,019(36,822)$ selected events in data in the muon (electron)+jets channel. From simulation, the

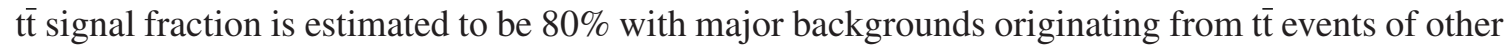
decay channels $(12 \%)$, single-top-quark events $(4 \%)$ and $\mathrm{W}+$ jets events $(2 \%)$. The background is subtracted from the selected events as estimated from the simulation.

The reconstruction of kinematic properties of the top-quark pair is performed with a kinematic fit constraining the reconstructed W-boson masses to $80.4 \mathrm{GeV}$ and requiring equal reconstructed top- and antitop-quark masses.

The reconstructed distributions are corrected for detector effects such as inefficiencies and migration using a regularised unfolding. The detector response matrix is estimated from the MADGRAPH [8] t $\mathrm{t}$ signal simulation with data-driven corrections for the trigger and lepton-selection efficiencies. For the $\mathrm{t} \overline{\mathrm{t}}$-system and top-quark quantities, the distributions are unfolded to parton level and extrapolated to the full phase space, whereas the lepton and b-jet distributions are unfolded to the stable-particle level in the visible phase space.

The differential cross sections are finally normalised using the in-situ determined inclusive cross section, reducing systematic uncertainties that are correlated across all bins. The remaining uncertainties of between 3 and 10\% (depending on the exact quantity and bin) are dominantly from model variations such as the MADGRAPH matching scale and the hard-scattering scale $Q^{2}$.

\section{Results and Conclusions}

As an example, the resulting normalised differential cross sections for the top-quark $p_{\mathrm{T}}$ and $\mathrm{t} \overline{\mathrm{t}}$ invariant mass distribution are shown in Fig. 1. The distributions of further quantities (rapidity of 

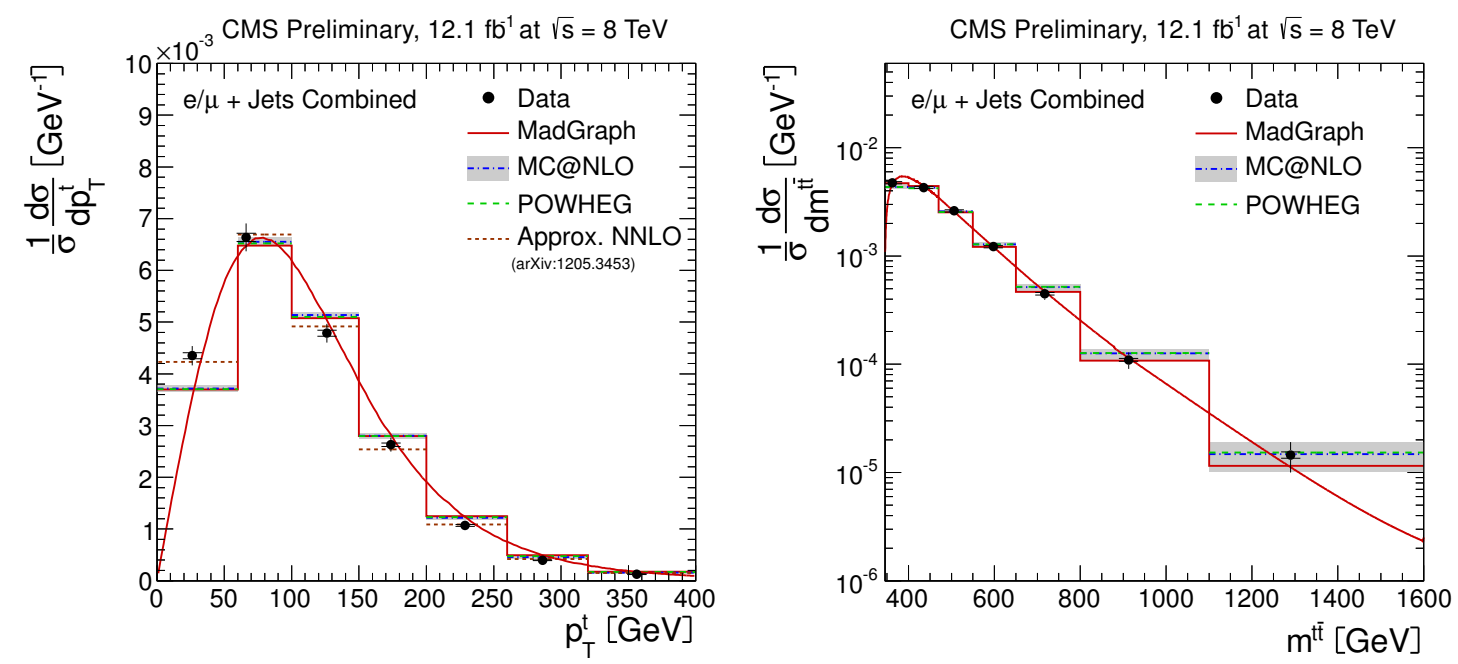

Figure 1: Normalised differential tt cross sections as a function of the $p_{\mathrm{T}}$ of the top quark (left) and the invariant mass of the t⿱t $\mathrm{system}$ (right).

the top quark and the $t \bar{t}$ system; $p_{\mathrm{T}}$ of the $\mathrm{t} \overline{\mathrm{t}}$ system, the charged lepton and $\mathrm{b}$ jets; and pseudorapidity of the charged lepton and $b$ jets) are shown in Ref. [6].

The results are compared to different model predictions from Monte-Carlo event generators. The top-quark quantities are additionally compared to approximate next-to-next-to-leading-order (NNLO) calculations [9]. Generally, a good agreement is observed between data and the various SM predictions. The top-quark- $p_{\mathrm{T}}$ distribution is measured slightly softer than the Monte-Carlo predictions, but the higher-order approximate NNLO calculation describes the data well. The results agree with those obtained in the dileptonic channel [7] and show qualitatively the same agreement between data and predictions as the ones at $7 \mathrm{TeV}$ [3].

To conclude, top-quark-pair physics seems to be well-described within the SM and no signs of new physics are observed at the current level of precision.

\section{References}

[1] CMS Collaboration, JINST 3 (2008) S08004.

[2] ATLAS Collaboration, JINST 3 (2008) S08003.

[3] CMS Collaboration, Eur. Phys. J. C73 (2013) 2339.

[4] ATLAS Collaboration, Eur. Phys. J. C73 (2013) 2261.

[5] ATLAS Collaboration, ATLAS-CONF-2013-099.

[6] CMS Collaboration, CMS-PAS-TOP-12-027.

[7] CMS Collaboration, CMS-PAS-TOP-12-028.

[8] J. Alwall et al., JHEP 06 (2011) 128.

[9] N. Kidonakis, arXiv:hep-ph/1205.3453. 